\title{
海上张力腿平台张力筋腱连接器构型设计与优化
}

\author{
王立权 ${ }^{1}$ 李松羽 ${ }^{2}$ 弓海霞 ${ }^{1}$ 刘杭杭 $^{1}$ 张璐瑶 $^{3}$ \\ (1. 哈尔滨工程大学机电工程学院 哈尔滨 150001; \\ 2. 中国船舶科学研究中心上海分部 上海 200011; \\ 3. 中国船舶重工集团公司第 711 研究所 上海 201108)
}

\begin{abstract}
摘要: 张力腿平台通过张力筋腱与海底连接, 从而约束了张力腿平台升沉方向上运动, 并在一定范围内限制其水平方向的运 动, 这种动态载荷是由张力筋腱及其连接器承担的, 因此张力筋腱的柔性连接成为张力腿平台研发过程中的关键研究内容之 一。目前国内尚缺乏对张力筋腱连接器的研究, 因此详细研究了张力腿平台张力筋腱连接器构型, 建立张力筋腱顶部连接器 锁紧机构的力学模型, 通过响应面法对锁定机构的牙型角、齿根厚度、齿顶厚度和分瓣螺母底面夹角等关键参数进行优化, 以降低最大等效应力, 延长连接器的疲劳寿命, 提高连接稳定性。研制张力筋腱底部连接器缩比原理样机, 完成张力筋腱底 部连接器陆地与水池安装试验, 通过试验验证张力筋腱底部连接器构型的合理性。结果表明, 经过优化后的顶部连接器性能 有明显提升, 与优化前相比, 最大等效应力降低了 $26.5 \%$, 而接触反力增加了 $37.4 \%$ 。底部连接器构型合理, 均可以完成张 力筋腱的连接要求。
\end{abstract}

关键词: 张力腿平台; 张力筋腱; 顶部连接器; 底部连接器; 多目标优化

中图分类号: TE937

\section{Configuration Design and Optimization of TLP Tendon Connectors}

\section{WANG Liquan $^{1}$ LI Songyu ${ }^{2}$ GONG Haixia ${ }^{1}$ LIU Hanghang ${ }^{1}$ ZHANG Luyao ${ }^{3}$}

(1. School of Mechanical and Electrical Engineering, Harbin Engineering University, Harbin 150001;

2. Shanghai Branch of China Ship Science Research Center, Shanghai 200011;

3. Shanghai Marine Diesel Engine Research Institute, Shanghai 201108)

\begin{abstract}
Tension leg platform(TLP) is connected to the seabed by tendons. Thus the movement of TLP in the heave direction is constrained and the horizontal direction movement is limited within a certain range. Then great dynamic tension loads will be supported by the tendons and the connectors. Therefore, the tendon connection becomes one of the key technologies in the TLP R\&D process. For lack of relevant studies on the tendon connector, the structural configuration of the tendon connector is studied. The mechanical model of the locking mechanism of the tendon top connector(TTC) is established. The response surface methodology is used to optimize the four parameters of the angle of the tooth, the thickness of the root, the thickness of the tooth tip and the angle of the bottom of the slip nut of the top connector locking structure to reduce the maximum equivalent stress, extend the fatigue life of the connection structure and improve the connection stability. The tendon bottom connector prototype(TBC) is developed. The onshore and pool installation test of the tendon is proposed. The rationality of the TBC structure configuration is verified by the test. The results show that the optimized performance of TTC is significantly improved. The maximum equivalent stress was reduced by $26.5 \%$, while the contact reaction increased by $37.4 \%$. The TBC configuration is reasonable and the connection requirements of tendons can be completed.
\end{abstract}

Key words: tension leg platform; tendon; tendon top connector; tendon bottom connector; multi-objective optimization

\section{0 前言}

张力腿平台是一种深海海洋结构物, 主要用

* 国家自然科学基金资助项目(51779064)。20180921 收到初稿, 20190320 收到修改稿
于深海钻井及采油。张力腿平台通过张力筋腱与 海底桩基连接, 张力筋腱通过顶部连接器与张力 腿平台底部浮箱连接, 通过底部连接器与海底桩 基连接 ${ }^{[1]}$ 。张力腿平台的主要特点是其浮箱提供的 浮力远大于平台自身的重力, 因此设计有张力筋 腱系统为张力腿平台提供垂直系泊功能 ${ }^{[2]}$ 。为了弥 
补多余浮力, 当平台正常工作时张力筋腱内部会 一直处于正拉力状态, 这一特点使张力腿平台在 水中的状态类似于倒置的钟摆 ${ }^{[3]}$, 此时平台的升沉 方向运动(垂荡、横摇和纵摇)受到张力筋腱的限 制, 但是在水平方向(艄摇、横荡、纵荡)上允许有 一定范围内的运动, 因此在张力筋腱及其连接器 内会产生巨大的动态载荷。由于张力腿平台对重 力的敏感性, 工作水深对平台排水量影响较大, 继而影响张力筋腱的尺寸, 而张力筋腱的尺寸会 影响张力筋腱连接器的尺寸及性能 ${ }^{[4]}$ 。PALLINI 等 ${ }^{[5-6]}$ 研究了 Jolliet 平台的张力筋腱顶部与底部连 接器, 概述了该连接器的设计、分析和测试过程 以及在位安装方法。通过有限元分析, 得出了影 响张力筋腱连接器应力集中的参数, 主要包括平 行卡齿牙型角、分瓣螺母底部偏角等。BOYUM ${ }^{[7]}$ 使用 ADINA 商业有限元软件对张力筋腱顶部连接 器进行了优化设计, 文中详细的描述了有限元分 析过程, 根据线弹性和弹塑性分析可知, 顶部连 接器中的载荷传递分布、应力集中以及疲劳寿命 受平行卡齿加工公差的强烈影响。但是限于当时 的计算机计算能力, 该仿真网格划分较为粗䊁, 结果误差较大。2013 年, Hutchinson 公司的张力 筋腱底部连接器首次应用在张力腿平台上。 D'ANGELO 等 ${ }^{[8]}$ 对该底部连接器进行了概述, 并 详细描述了 DNV 的技术认证过程及结果。国内目 前尚没有针对张力筋腱连接器进行相关研究。本 文详细研究了张力筋腱顶部与底部连接器构型; 通过响应面法对顶部连接器锁定机构进行了优 化, 降低了局部应力集中, 增强了锁紧性能。研 制了张力腿平台张力筋腱底部连接器原理样机, 通过水池试验研究了张力筋腱的安装过程, 验证 了张力筋腱底部连接器构型的合理性。

\section{1 张力筋腱连接器构型研究}

\section{1 张力腿平台张力筋腱连接器载荷分析}

张力腿平台是一种海上石油平台, 其特点是 通过一种被称为张力筋腱的钢管与海底桩基相 连, 如图 1 所示, 因此同时具有刚性与顺应性平 台的特点。

张力腿平台在工作期间受到各种环境力(包括 海流、海浪、风等)的作用, 继而发生横荡、纵荡、 垂荡、横摇、纵摇和艄摇等运动, 如图 2 所示。随 着平台的运动, 会对张力筋腱上施加弯矩及压力载 荷, 为了保证结构不发生破坏, 需要分析计算张力 腿平台在不同工况下受到的载荷。

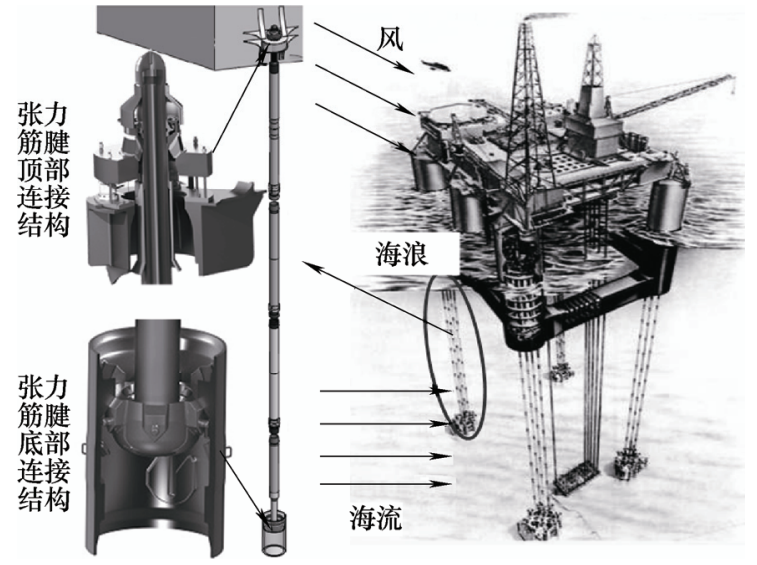

图 1 张力腿平台与张力筋腱连接结构

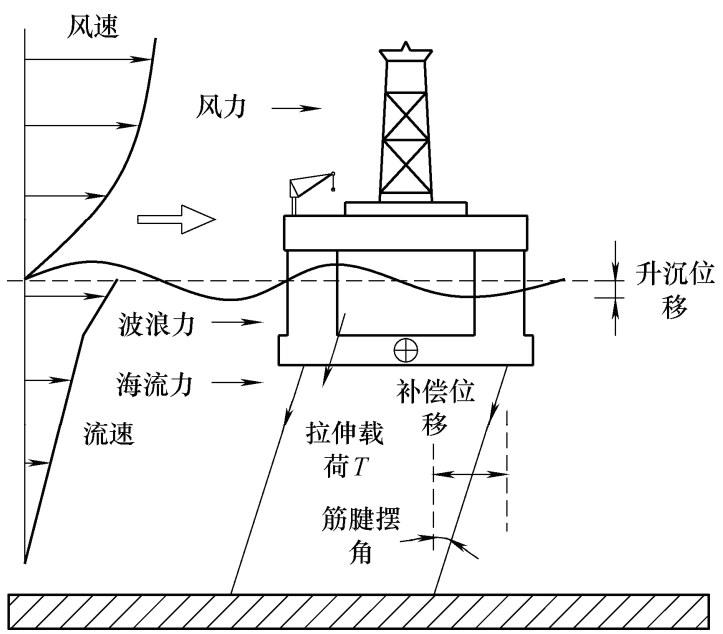

图 2 张力腿平台受载荷情况

除了需要承受巨大载荷外, 张力筋腱连接器还 需要方便快速安装, 为了满足这些要求, 在设计张 力筋腱连接器的过程中, 提出了不同于法兰或螺纹 连接的特殊形式。

\section{2 顶部连接器构型研究}

早期的张力筋腱顶部连接器一般采用传统的螺 纹连接结构, 由于筋腱尺寸较大, 螺母的转动使安 装过程较为复杂, 因此提出了一种新的连接构型。 图 3 所示为典型的张力筋腱顶部连接器锁紧机构, 主要由分瓣螺母、支撑环、安装工具、柔性体组成。 在张力筋腱顶部有一段特殊处理的钢管称为长度调 整段, 为了使分瓣螺母可以抱紧张力筋腱, 在该段 外部有平行卡齿。为了使分瓣螺母可以根据需要抱 紧与松开, 因此在分瓣螺母外侧设计了安装工具。 安装工具上有六个液压缸, 每个液压缸对应一个分 瓣螺母, 在液压缸伸缩杆底部有卡扣, 卡扣勾住分 瓣螺母外侧使其可以上下移动。需要锁紧顶部连接 器时, 液压缸杆伸长, 分瓣螺母下降; 需要解锁时, 缸杆收缩, 分瓣螺母上升。通过设计平行卡齿牙型 角, 使张力筋腱仅允许向上提起。当张力筋腱有下 落的趋势时, 分瓣螺母受到径向对中力, 会将张力 
筋腱抱紧, 顶部连接器不会断开。由于顶部连接器 的平行卡齿结构, 无法通过涂漆的方式进行防腐处 理, 因此通常在顶部连接器外安装防腐罩, 防腐罩 内充满防腐液。

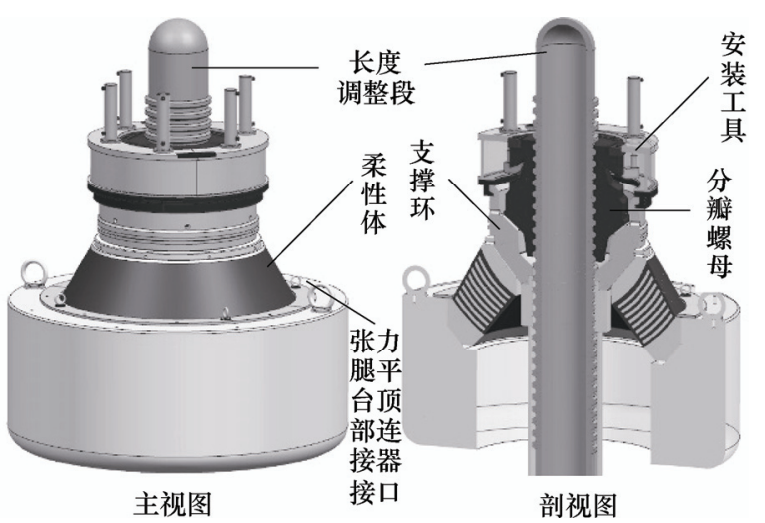

图 3 顶部连接器示意图

图 3 所示的顶部连接器具有 6 块分瓣螺母, 其 中一块分瓣螺母外形如图 4a 所示。在连接张力筋腱 前, 首先通过安装工具将所有分瓣螺母固定在长度 调整段与支撑环之间。分瓣螺母底部设计有斜面, 其倾斜角度与支撑环顶部的斜面一致, 当分瓣螺母 处于自由状态时, 其受到的重力通过斜面分解为向 张力筋腱轴心夹紧的分力, 从而使分瓣螺母与支撑 环之间的接触面贴合得更紧密。通过对分瓣螺母底 面偏角和平行卡齿牙型角进行参数设计与优化, 可 以使顶部连接器具有自锁能力。

图 4b 所示为顶部连接器的安装工具, 安装工具 上安装有六个液压缸, 通过甲板液压源驱动。图 5 所示为其液压原理图, 该系统采用变量泵调速, 使 用一个三位四通电磁换向阀, 可以同步控制液压缸 的运动。液压缸带负载工作时, 通过调整变量原排 量调整液压缸的行进速度, 通过主溢流阀调节最大 工作压力, 主溢流阀由远程先导溢流阀调定。

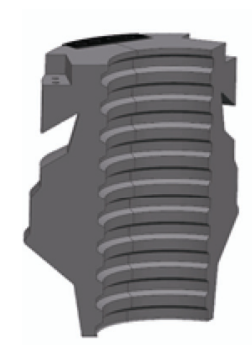

(a) 分瓣螺母

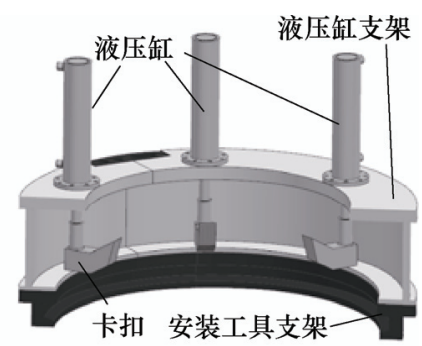

(b) 安装工具
图 4 顶部连接器组件

张力腿平台是一种半刚性半顺应性平台, 由于 张力筋腱连接器在转动自由度上具有的柔性, 为平 台提供了顺应性。为了实现转动自由度上的柔性连 接, 在张力筋腱连接器中安装了柔性部件。使用橡 胶材料可以获得转动柔性, 使用金属材料则可以满
足压缩刚度。由于需要承受巨大的拉伸与弯曲载荷, 为了同时提供转动柔性及压缩刚性, 在顶部连接器 中安装了柔性体 ${ }^{[9]}$ ，该元件使用金属和橡胶层叠材 料加工得到。
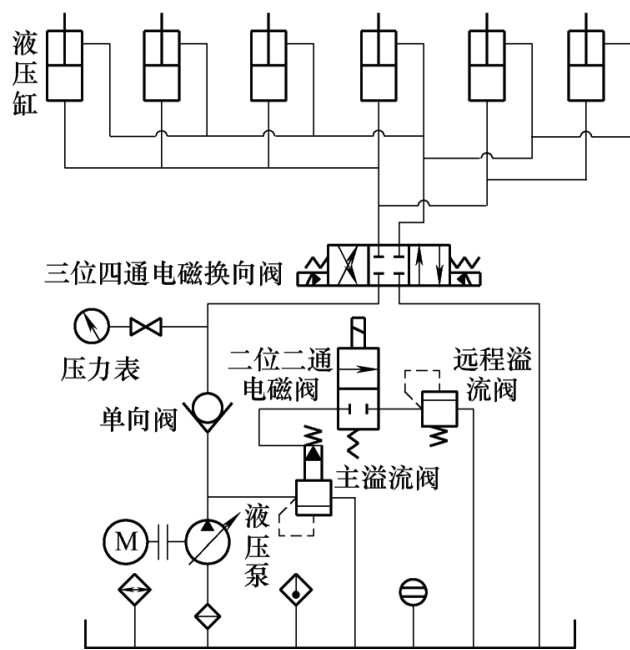

图 5 安装工具液压系统原理图

如图 6 所示为顶部连接器用柔性体, 该元件采 用球台结构, 通过粘合剂将橡胶片与钢板初步固定, 最后硫化完成最终定型，柔性体同时具有金属与橡 胶材料的优点。柔性体内部的橡胶片与金属板具有 相同的球心, 橡胶片夹在金属板之间形成层叠结构, 橡胶的柔性使张力筋腱摆动时端部不会发生应力集 中, 实现了柔性连接, 而钢板则使端部具有较大的 轴向压缩刚度。

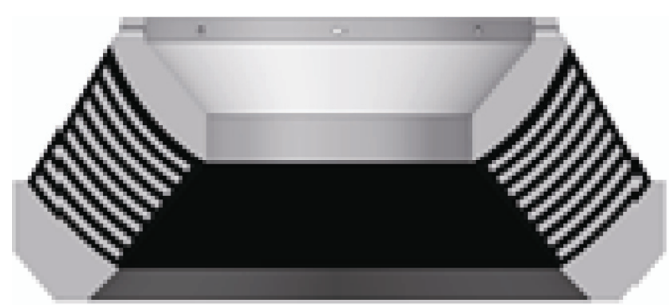

图 6 张力筋腱顶部连接器柔性体

\section{3 底部连接器构型研究}

由于张力腿底部连接器在海底工作, 采用传统 连接方式需要复杂的安装过程, 因此提出了一种新 的连接形式。如图 7 所示为典型的旋转锁紧式张力 筋腱底部连接器, 此类连接器由公接头与接收器组 成。其中公接头由载荷环、柔性体和导向环组成, 载荷环同时也作为公接头的顶部端盖, 为了承受拉 伸与转动载荷, 载荷环内部也安装有柔性体, 由于 张力筋腱两端受到的载荷不完全相同, 因此两种连 接器中的柔性体刚度不同, 需要单独设计。在载荷 环外侧均有分布有 6 个承载凸缘，承载凸缘顶部也 设计有斜面，承载凸缘重要用于承受筋腱传递的载 荷。导向环同时也作为公接头的底部端盖, 导向环 
外侧均有分布有 6 个导向凸缘, 与承载凸缘的径向 位置相同。在公接头进入接收器的过程中, 导向凸 缘在接收器上的导向块间隙中移动, 使公接头转动。

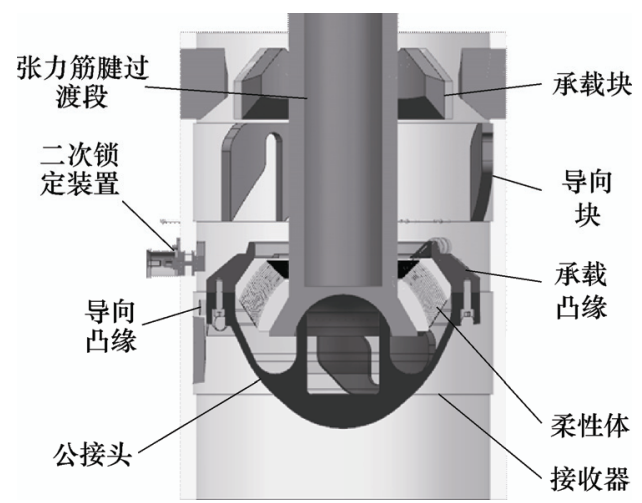

图 7 张力筋腱底部连接器与载荷分布

底部连接器的工作原理与圆珠笔类似, 如图 $8 \mathrm{a}$ 所示。当张力腿平台充入压载水从而下沉, 张力筋 腱带动公接头进入接收器, 导向凸缘受到接收器底 部导向块斜面的作用, 使公接头顺时针旋转; 当公 接头完全降落到接收器导向块以下时, 平台排空压 载水, 张力筋腱带动公接头上升, 导向凸缘受到接 收器顶部导向块斜面作用, 使公接头逆时针旋转, 直到承载凸缘与承载块完全接触, 底部连接器完成 锁定。根据该原理可知, 当底部连接器承受正向拉 力时, 连接器连接会越发稳定; 相反, 若张力腿平 台发生向下运动时, 底部连接器有随时断开的危险, 即该连接器不允许出现压缩载荷。

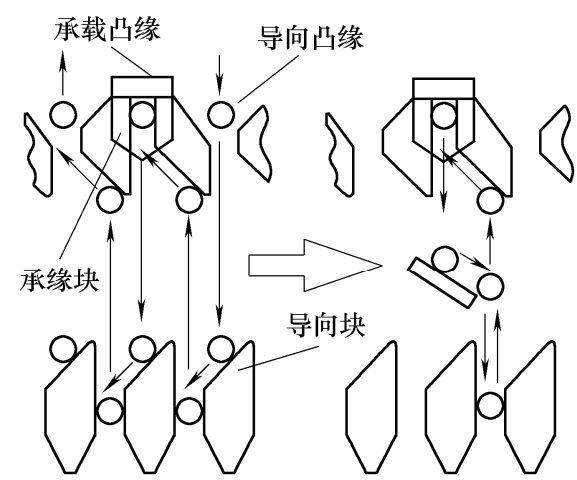

(a) 副锁定装置开启前 (b) 副锁定装置开启后

图 8 底部连接器工作原理

由于底部连接器的这一缺点曾经造成了重大事 故, 2005 年在墨西哥湾出现了特大型台风Rita过境, 台风造成的巨大海浪使当时工作在这一片海域的 Typhoon 张力腿平台出现了大幅度升沉运动, 最终 造成了张力筋腱底部连接器脱开事故, 继而使平台 倾覆，该事故造成了上亿美元的损失 ${ }^{[10]}$ 。为了克服 该缺点, 本文提出了底部连接器二次锁定的概念, 并设计了二次锁定装置。该装置主要由挡块、固定
底、TDU 把手组成，如图 9 所示。其工作原理如图 $8 \mathrm{~b}$ 所示, 当底部连接器完成锁定后, ROV 转动 TDU 把手, 通过丝杜螺母机构使挡块伸出, 一旦底部连 接器出现负向拉力, 二次锁定装置会将公接头导向 回到载荷块, 张力筋腱不会发生脱离。由于该装置 仅起导向作用，因此基本不会出现强度问题。

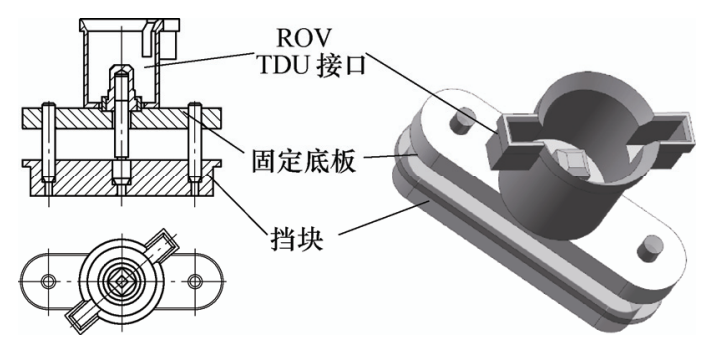

图 9 二次锁定装置结构图

\section{2 顶部连接器锁定机构多目标优化}

\section{1 顶部连接器锁定机构力学研究}

根据第 1.2 节可知, 为了使顶部连接器具有自 锁能力, 需要对分瓣螺母进行受力分析, 从而优化 分瓣螺母的底面偏角及平行卡齿牙型角。将连接器 的自锁能力定义为: 当张力筋腱受到向下拉力时, 分瓣螺母有向张力筋腱长度调整段中心轴合拢的趋 势。对此时的分瓣螺母进行受力分析，分瓣螺母受 到的载荷包括: 支撑环斜面上的支撑力 $F_{\mathrm{s}}$ 、张力筋 腱长度调整段对平行卡齿的压力 $F_{\mathrm{n}}$ 和摩擦力 $F_{\mathrm{f}}$ 以 及分瓣螺母自身的重力 $G$ 。由于长度调整段上的拉 力远大于分瓣螺母的重力, 因此忽略该重力。如图 10 所示, 对分瓣螺母所受载荷进行分解坐标分解, 得到 $x 、 y$ 方向上的分力分别为

$$
\left\{\begin{array}{c}
F_{\mathrm{x}}=F_{\mathrm{s}} \sin \alpha-F_{\mathrm{c}} \sin \beta- \\
F_{\mathrm{f}} \cos \alpha-F_{\mathrm{f}}^{\prime} \cos \beta-F_{\mathrm{n}} \\
F_{\mathrm{y}}=F_{\mathrm{z}} \cos \alpha-F_{\mathrm{c}} \cos \beta+ \\
F_{\mathrm{f}} \sin \alpha+F_{\mathrm{f}}^{\prime} \sin \beta
\end{array}\right.
$$

式中, $F_{\mathrm{f}}=\mu_{1} F_{\mathrm{s}}, F_{\mathrm{f}}^{\prime}=\mu_{2} F_{\mathrm{c}}$ 分别为分瓣螺母受到的支 撑环与张力筋腱长度调整段的总摩擦力; $\mu_{1}$ 为支撑 环和分瓣螺母的动摩擦因数, $\mu_{2}$ 为分瓣螺母和张力 筋腱长度调整段间的动摩擦因数。

当分瓣螺母具有自锁能力时, 应有 $F_{\mathrm{n}} \geqslant 0$, 当分 瓣螺母在准静力平衡状态下, 可以得到 $\alpha$ 与 $\beta$ 具有 如下关系

$$
\left\{\begin{array}{l}
F_{\mathrm{c}}\left(\sin \beta+\mu_{2} \cos \beta\right) \leqslant F_{\mathrm{s}}\left(\sin \alpha-\mu_{1} \cos \alpha\right) \\
F_{\mathrm{c}} \frac{\cos \beta-\mu_{2} \sin \beta}{\cos \alpha+\mu_{1} \sin \alpha}=F_{\mathrm{s}}
\end{array}\right.
$$




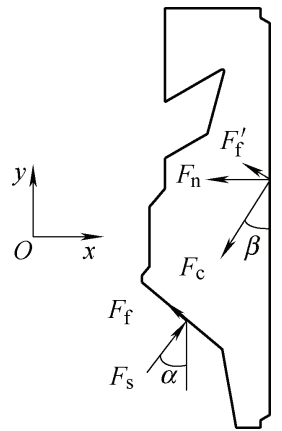

图 10 分瓣螺母受力分析

由于 $\alpha \in(0, \pi / 2)$, 则方程式(2)中的第二个方程 可以简化为

$$
\frac{\left(\cos \beta-\mu_{2} \sin \beta\right)\left(\sin \alpha-\mu_{1} \cos \alpha\right)}{\left(\sin \beta+\mu_{2} \cos \beta\right)\left(\cos \alpha+\mu_{1} \sin \alpha\right)} \geqslant 1
$$

顶部连接器一般采用不锈钢, 防腐液对金属间具 润滑作用, 润滑后的钢与钢之间静摩擦因数一般为 0.16 , 即 $\mu_{1}=\mu_{2}=0.16$, 则在保证自锁的前提下, 根据 方程式(3)可以解得分瓣螺母底面偏角 $\alpha$ 的取值范围

$$
\frac{\pi}{10}+\beta \leqslant \alpha<\frac{\pi}{2}
$$

\section{2 优化有限元仿真模型}

根据海上施工需求, 顶部连接器对重量和大小 非常敏感。顶部连接器在工作期间随时承受不均匀 载荷, 并且在平行卡齿的根部由于设计及加工的问 题, 会产生较高的应力集中系数(SCF), 因此需要对 连接器锁紧机构的关键参数进行优化。

进行有限元分析前首先对模型进行简化, 省略 其中一些不重要的部分, 防止参数变化可能产生的 网格错误。由于扭矩施加在连接器的中面上, 因此 建立 $1 / 2$ 平面对称有限元模型。连接器主要由不锈 钢制造, 因此采用线弹性本构模型, 其弹性模量 $E=2.1 \times 10^{5} \mathrm{MPa}$, 泊松比 $v=0.3$ 。为防止可能发生 的剪切锁定问题, 采用一阶缩减单元, 同时该单元 也较为适用于接触分析。有限元模型网格的总体尺 寸设置为 $20 \mathrm{~mm}$, 为了保证分析精确性, 对平行卡 齿部分的网格进行局部细化, 如图 11 所示。在支撑

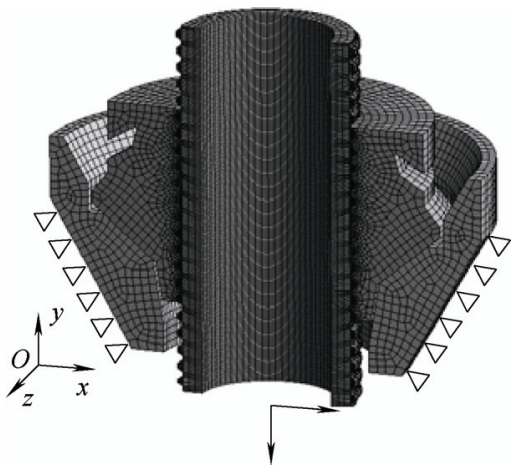

图 11 优化模型的有限元网格
环上底部斜面施加固定约束, 在张力筋腱底部施加 大小为 $4.9 \times 10^{7} \mathrm{~N}$ 的向下拉伸载荷以及 $3.3 \times 10^{6} \mathrm{~N}$ 的水平拉伸载荷, 在对称面上施加对称约束。在张 力筋腱和分瓣螺母间、分瓣螺母和支撑环间施加摩 擦接触，摩擦因数均为 0.15 , 分瓣螺母之间施加无 摩擦接触。

\section{3 优化目标与优化变量}

顶部连接器锁紧机构的关键参数优化目标主要 包括以下内容。

（1）降低锁紧机构极限应力, 提高顶部连接器 极限承载能力。

（2）最小化平行卡齿根部的应力集中，延长顶 部连接器的疲劳寿命。

(3) 提高分瓣螺母底部斜面与支撑环顶部斜面 间的接触压力, 提升顶部连接器的自锁能力。

使用哈尔滨工程大学材化学院自主研发的 $1 \mathrm{MnCrMoNi} 2 \mathrm{Cu}$ 高强度合金钢 ${ }^{[11]}$ 加工本文研究的 张力腿平台使用顶部连接器锁紧机构, 该合金钢的 拉伸屈服强度 $\sigma_{\mathrm{s}} \approx 1170 \mathrm{MPa}$, 因此顶部连接器锁紧 机构优化条件之一为最大等效应力小于 $1170 \mathrm{MPa}$ 。

由于顶部连接器采用正梯形平行卡齿, 通过 确定其牙型角 $\beta$ 、齿根厚度 $b_{1}$ 和齿顶厚度 $b_{3}$ 即可 以确定平行卡齿的形状。根据第 2.1 节的分析可 知, 平行卡齿的自锁定性还与分瓣螺母底面夹角 $\alpha$ 有关，因此对上述四个参数进行优化。节距与齿 根厚度会影响平行卡齿的承载能力, 牙型角会影 响平行卡齿根部的应力集中，过大的牙型角会影 响锁定性能, 综合考虑后将以上三种参数的优化 范围确定为

$$
\left\{\begin{array}{l}
36 \mathrm{~mm} \leqslant b_{1} \leqslant 44 \mathrm{~mm} \\
18 \mathrm{~mm} \leqslant b_{3} \leqslant 22 \mathrm{~mm} \\
25^{\circ} \leqslant \beta \leqslant 35^{\circ}
\end{array}\right.
$$

结合方程式(4)可知, 分瓣螺母底面夹角 $\alpha$ 应至 少大于等于 $43^{\circ}$, 由于 $\alpha$ 过大, 为了保证结果完整 性会导致分瓣螺母过长, 因此综合考虑后确定了 $\alpha$ 的优化范围为

$$
43^{\circ} \leqslant \alpha \leqslant 58^{\circ}
$$

\section{4 顶部连接器锁定机构优化结果}

使用响应面优化方法及 MOGA 算法对模型进 行优化分析 ${ }^{[12-13]}$, 该方法主要用于设计敏感度信 息难以计算或者具有噪声函数使敏感度信息不可 靠的情况 ${ }^{[14-17]}$ 。两两建立一组建立上述四个参数 的响应平面, 通过观察图 12 和图 13 所示响应面, 确定不同参数组合对最大等效应力和接触反力的 影响。 
对比图 $12 \mathrm{a} \sim 12 \mathrm{c}$ 可以发现, 随着平行卡齿的齿 顶厚增加, 顶部连接器锁定机构的最大等效应力近 似抛物线变化; 当齿顶厚 $b_{3}=19.6 \mathrm{~mm}$ 时, 最大等效 应力最小。对比图 $12 \mathrm{c} \sim 12 \mathrm{e}$ 可以发现, 随着平行卡 齿的齿根厚增加, 最大等效应力近似 $\gamma$ 形曲线变化; 当齿根厚 $b_{1}=40 \mathrm{~mm}$ 时, 最大等效应力达到极小值。

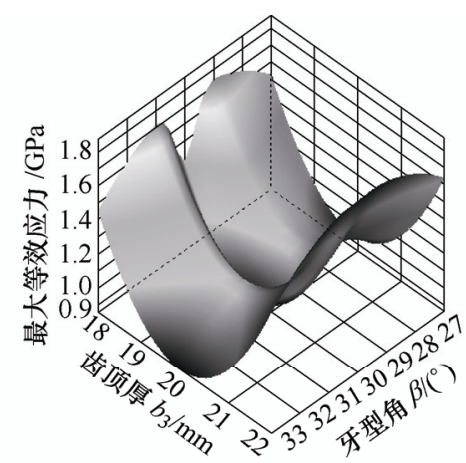

(a) 齿顶厚与牙型角

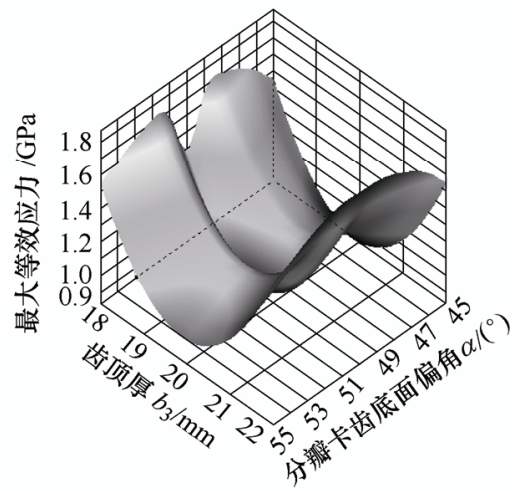

(h) 齿顶厚与分㦚卡齿底面偏角

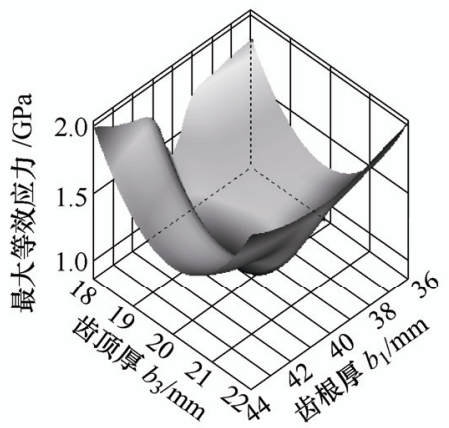

(c) 齿顶厚与齿根厚

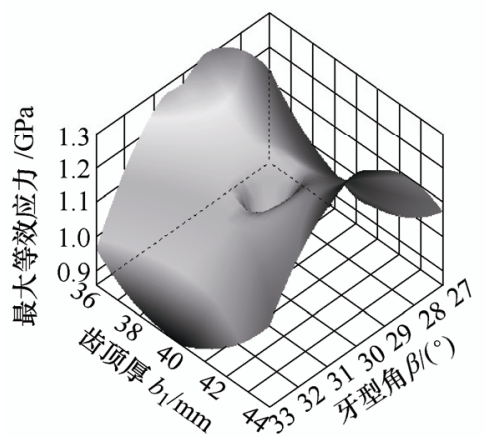

(d) 齿根厚与牙型角

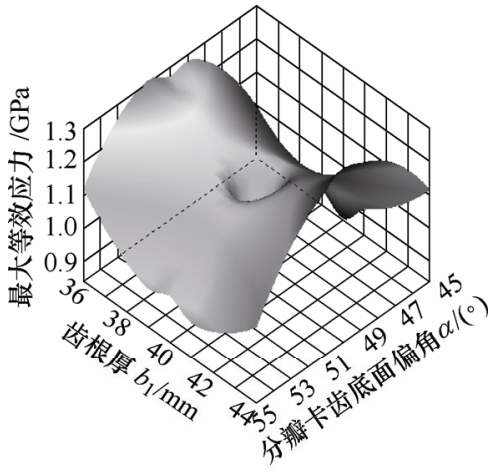

(e) 齿根厚与分㦚卡齿底面偏角

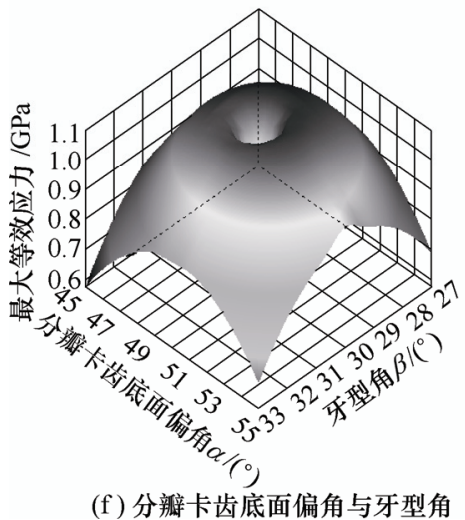

图 12 最大等效应力响应面

对比图 12a、12d、12f 可以发现，随着牙型角增加， 最大等效应力的变化曲线近似 $\mathrm{m}$ 型, 当牙型角 $\beta=29.1^{\circ}$ 或 $31.1^{\circ}$ 时, 最大等效应力达到极大值; 当牙型角 $\beta=30^{\circ}$ 时, 最大等效应力达到极小值; $\beta$ 最大或最小时，最大等效应力最小。对比图 $12 \mathrm{~b}$ 、 $12 \mathrm{e} 、 12 \mathrm{f}$ 可以发现, 分瓣螺母底面偏角增大时, 最大等效应力的变化曲线也近似 $\mathrm{m}$ 型, 当分瓣螺 母底面偏角 $\alpha=48.3^{\circ}$ 或 $51.7^{\circ}$ 时, 最大等效应力达 到极大值; 当分瓣螺母底面偏角 $\alpha=50{ }^{\circ}$ 时, 最大 等效应力达到极小值; $\alpha$ 最大或最小时，最大等效 应力最小。

对比图 13a $13 \mathrm{c}$ 可以发现, 随着齿顶厚增加, 接触反力变化曲线近似抛物线, 当 $b_{3}=20.2 \mathrm{~mm}$ 时, 接触反力最大, $b_{3}$ 最大或最小时接触反力最小。对 比图 13e $13 \mathrm{e}$ 可以发现, 齿根厚增大时, 接触反力 变化曲线也近似抛物线, 当齿根厚 $b_{1}=39.8 \mathrm{~mm}$ 时, 接触反力最大, $b_{1}$ 最大或最小时接触反力最小。对 比图 13a、13d、13f 可以发现, 牙型角增加时, 接 触反力变化曲线近似 $\mathrm{W}$ 形, 当 $\beta=28.3^{\circ}$ 或 $31.8^{\circ}$ 时, 接触反力达到极小值; 当 $\beta=30^{\circ}$ 时, 接触反力达到 极大值; $\beta$ 最大或最小时接触反力最大。对比图 13b、 $13 \mathrm{e} 、 13 \mathrm{f}$ 可以发现, 分瓣螺母底面偏角增大时, 接 触反力曲线也近似 $\mathrm{W}$ 形, 当 $\alpha=48.3^{\circ}$ 或 $51.7^{\circ}$ 时, 接触反力达到极小值; 当 $\alpha=50$ 时, 接触反力达到 
极大值, $\alpha$ 最大或最小时接触反力最大。

响应曲面法综合考虑目标函数及约束变量的要 求, 得到最优解如表 1 所示。与优化前的结果作比 较, 最大等效应力降低了 $26.5 \%$, 而接触反力增加 了 $37.4 \%$, 表明该优化显著提高了连接器的自锁性 能, 并降低了强度要求。

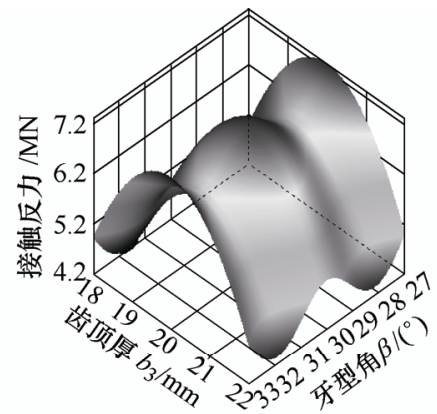

(a) 齿顶厚与牙型角

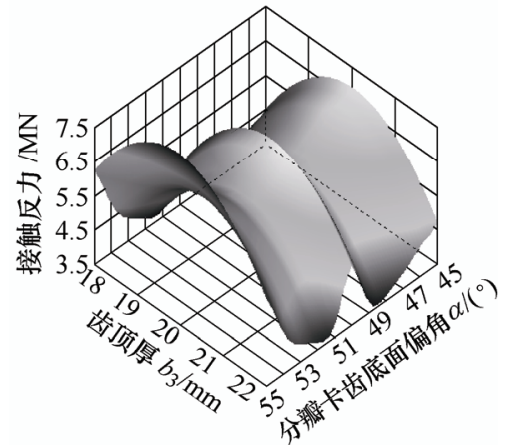

(b) 齿顶厚与分㦚卡齿底面偏角

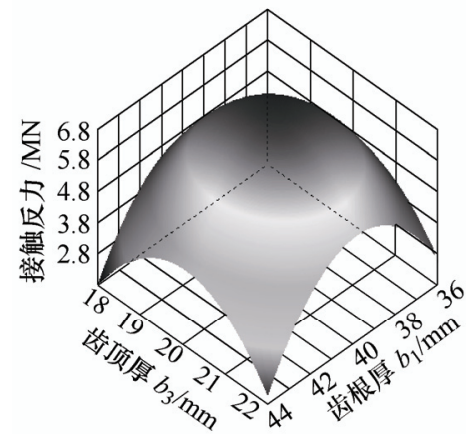

(c) 齿顶厚与齿根厚

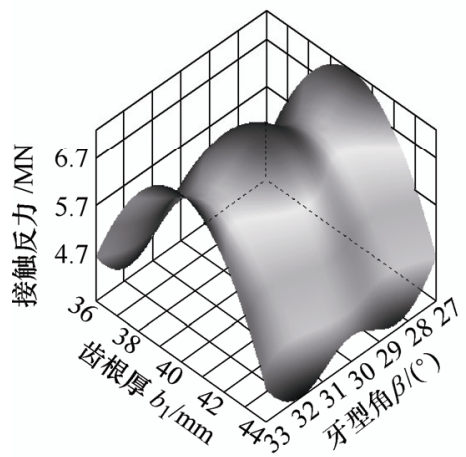

(d) 齿根厚与牙型角

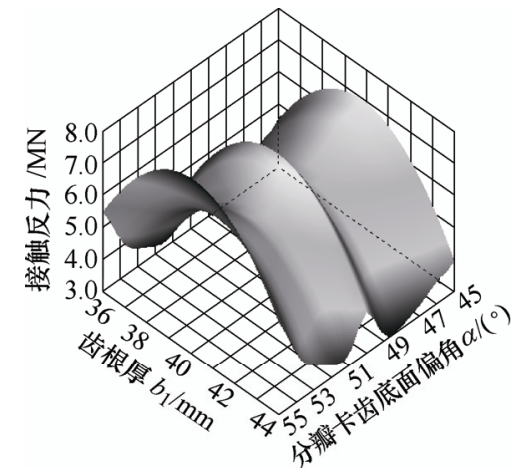

(e) 齿根厚与分㦚卡齿底面偏角

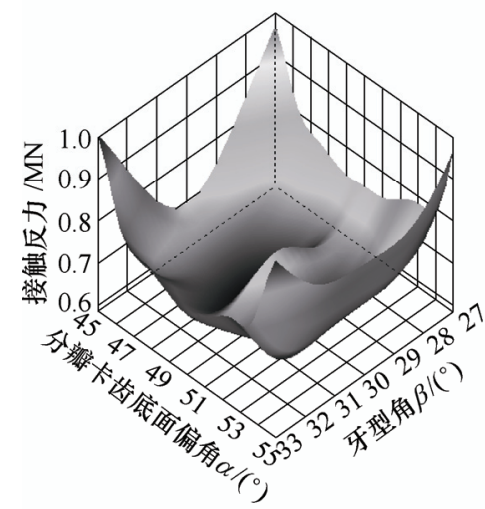

(f) 分瓣卡齿底面偏角与牙型角

图 13 接触反力响应面

表 1 锁定机构最优解

\begin{tabular}{cccccc}
\hline $\begin{array}{c}\text { 齿根厚度 } \\
b_{1} / \mathrm{mm}\end{array}$ & $\begin{array}{c}\text { 齿顶厚度 } \\
b_{3} / \mathrm{mm}\end{array}$ & $\begin{array}{c}\text { 夹角 } \\
\alpha /\left(^{(}\right)\end{array}$ & $\begin{array}{c}\text { 牙型角 } \\
\beta /\left(^{\circ}\right)\end{array}$ & $\begin{array}{c}\text { 最大等效应变/ } \\
\mathrm{MPa}\end{array}$ & $\begin{array}{c}\text { 接触反力/ } \\
\mathrm{N}\end{array}$ \\
\hline 39.97 & 19.97 & 55 & 33 & 660.79 & $1.0060 \times 10^{7}$ \\
\hline
\end{tabular}

图 14 所示为优化后的张力筋腱顶部连接器结 构图, 该顶部连接器可以承受最大 $40300 \mathrm{kN}$ 的轴 向拉力, 并允许发生 11.3 的偏角, 该设计基本达 到国际水平。
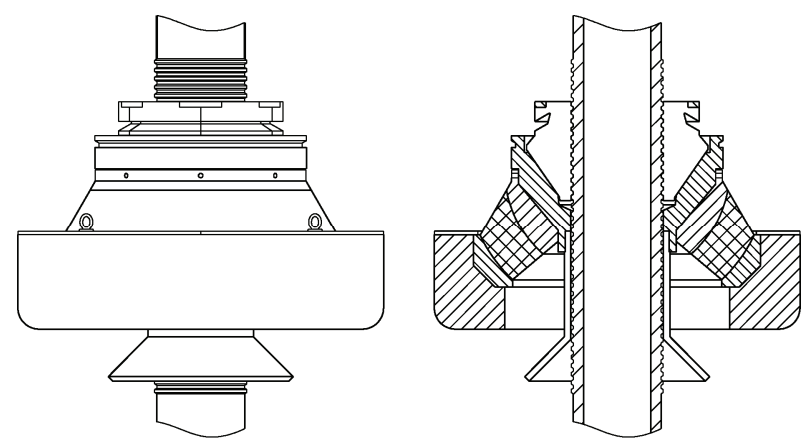

图 14 顶部连接器优化结果

3 底部连接器原理样机安装试验

\section{1 底部连接器原理样机研制}

为了验证张力筋腱底部连接器的连接形式, 本 文研制了如图 15 所示的底部连接器的缩比原理样 
机, 并开展水池试验。该原理样机的总体尺寸约为 $740 \mathrm{~mm} \times 560 \mathrm{~mm} \times 560 \mathrm{~mm}$ 。为了使公接头更容易 进入接收器, 在接收器原理样机的顶部设计了喇叭 口(在工程样机上, 该喇叭口安装在海底桩基上)。 由于原理样机为人工操作, 因此将工程样机中二次 锁定装置 ROV 接口改为一字形旋钮。在实际海上 施工时, 底部接收器固定在桩基上, 桩基通过打桩 锤固定在海底, 防止发生运动。为了增加接收器在 水池试验中的稳定性, 提高试验的安全性, 在接收 器底部焊接有配重板, 该配重板重量为 $1 \mathrm{t}$ 。
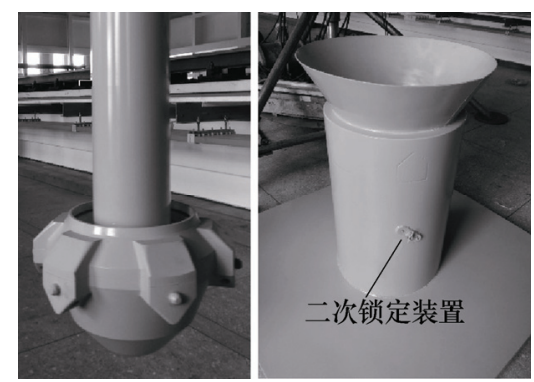

图 15 底部连接器原理样机

\section{2 底部连接器陆地安装试验}

在开展水池试验之前, 首先在陆地上开展了底 部连接器的功能性试验, 确定底部连接器设计的合 理性，同时也观察二次锁定装置是否可以实现预期 功能, 保证水池试验可以顺利完成。

由于底部连接器较重, 试验过程中需要吊装设 备, 因此该试验在设备加工厂内进行, 试验过程中 的温度为 $25{ }^{\circ} \mathrm{C}$ 。该原理样机包括底部配重的总重 量约为 $1.7 \mathrm{t}$, 在公接头顶部设计一段吊环, 通过吊 绳与吊车连接。底部连接器陆地试验主要包括连接 器锁定试验、二次锁定装置功能试验和解锁试验, 为排除可能出现的意外, 试验重复进行 5 次。

(1) 锁定试验: 试验前首先将二次锁定装置关 闭。使用吊车将底部连接器公接头运送到接收器顶 部, 使公接头缓慢下降, 落入接收器中, 由于有喇 叭口, 公接头不需要准确对中, 依旧顺利进入接收 器。当公接头底部接触到配重板后提升公接头, 运 动过程中观察公接头是否按照预想轨迹完成导向, 直至公接头的承载凸缘接触到接收器载荷块, 如图 16 所示。继续提升公接头, 接收器随公接头上升, 不发生脱离, 说明已完成锁定。
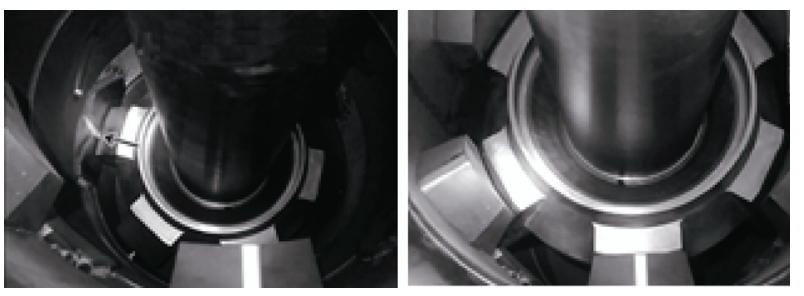

图 16 底部连接器安装阶段
(2) 解锁试验: 将接收器与公接头下方至吊绳 中没有拉力, 继续下放公接头, 观察公接头是否按 照预期路径运动至接触配重板, 上提公接头, 公接 头顺利脱离接收器, 完成移除, 如图 17 所示。
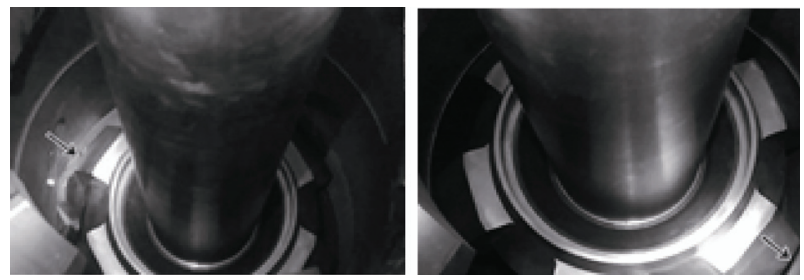

图 17 底部连接器移除阶段

(3) 二次锁定装置功能试验: 根据 API RP 2T 标准要求, 张力筋腱底部连接器需要可以承受千年 一遇的恶劣海况 ${ }^{[13]}$, 并保证不会断开, 二次锁定 装置可以实现该要求。首先根据锁定试验的步骤完 成连接底部连接器锁定, 人工转动接收器上的三个 二次锁定装置旋钮完成锁定，然后重复解锁试验步 骤, 发现无论下放几次, 公接头均无法脱离接收 器, 说明二次锁定装置工作正常, 如图 18 所示。
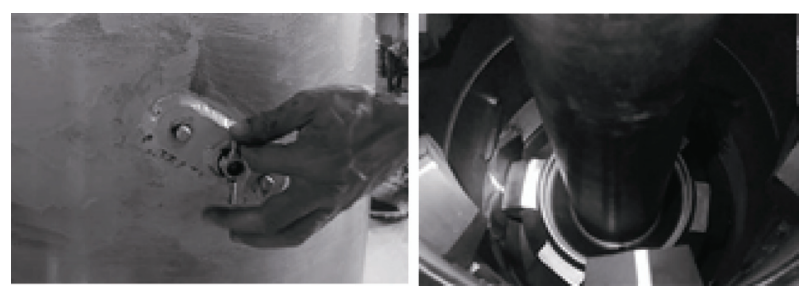

图 18 底部连接器工作阶段

重复上述试验共 5 次, 消除可能发生的意外。 发现试验中连接器均可以按照预定要求完成锁 定、解锁功能, 试验成功率 $100 \%$ 。试验过程中未 发生卡顿, 连接器外部结构没有发生破坏, 二次 锁定装置运行流畅, 可以起到预期效果。试验结 果显示该样机可以良好地完成试验流程, 可以用 于水池试验。

\section{3 底部连接器水池安装试验}

张力筋腱安装水池试验在哈尔滨工程大学 10 $m$ 深综合试验水池进行, 该试验使用 ZDKJ-A1 型井 下探测仪作水下视频监测, 其探头采用 304 全不锈 钢材料, 超广角镜头, 分辨率达到 $0.1 \mathrm{~mm}$, 采用 led 强光源、高清图像。主控制箱可配接计算机进行数 字化采集和存咜, 适用于各种复杂的对象和观测环 境。试验开始前, 将样机接头与接收器对接, 并利 用水池龙门吊车将样机放入水池中地势平坦区域, 其入水过程如图 19 所示。在公接头筋腱平滑处安装 有水下录像设备, 实时监测水下安装过程。由于二 次锁定试验需要潜水员配合, 本文仅完成了水下的 锁定与解锁试验。 

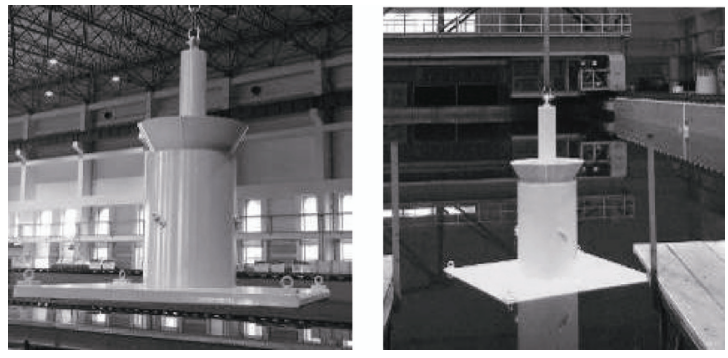

图 19 样机入水过程

(1) 锁定试验: 安装阶段的目的是对接连接器, 由吊机将接头吊入连接器, 下落至连接器最低处后 向上拉，完成安装，如图 20 所示。
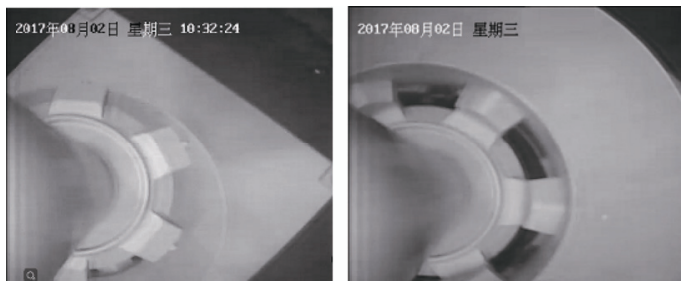

图 20 安装阶段示意图

(2) 解锁试验: 移除阶段的主要目的是解锁连 接器, 吊车带动接头下行至接收器底部后, 上提接 头即可脱出, 如图 21 所示, 完成移除。
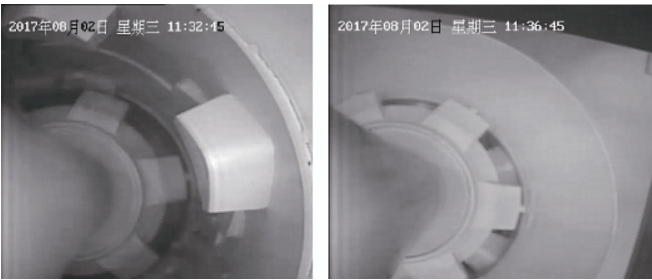

图 21 移除阶段示意图

同样重复上述试验共 5 次, 消除可能发生的意 外情况。试验过程中底部连接器均顺利完成了锁定 与解锁过程, 试验成功率 $100 \%$ 。试验过程中未发 生卡顿, 连接器外部结构没有发生破坏, 验证了底 部连接器试验样机的水下性能。

\section{4 结论}

(1) 张力筋腱顶部连接器由长度调整段、柔性 体、支撑环、安装工具和分瓣螺母组成。张力筋腱 底部连接器由张力筋腱过渡段、公接头、柔性体、 接收器, 可以完成张力筋腱与海底基础的快速连接 功能。

（2）对顶部连接器锁定机构中平行卡齿的牙型 角、齿根厚度、齿顶厚度和分瓣螺母底面夹角四个 变量进行优化。与优化前相比, 最大等效应力降低 了 $26.5 \%$, 而接触反力增加了 $37.4 \%$, 结果表明该 优化对顶部连接器性能有明显提升。
（3）根据筋腱底部连接器方案研制了缩比原理 样机，通过陆地和水池安装试验，验证其内部导向 结构的正确性、合理性，二次锁定装置在工作中的 功能表现良好, 安装过程顺利, 为后续研制提供有 价值的参考。

\section{参 考 文 献}

[1] DAVIES K B, SRIVAREERAT M. Morpeth SeaStar: foundation and tendon system $[\mathrm{C}] / /$ Proceedings of the Annual Offshore Technology Conference, Offshore Technology Conference. Houston, Texas: OTC, 1999: $1-11$.

[2] SINHA P, DATTA N, KANNAMWAR A. Vortex-induced vibration of a tension leg platform tendon: Multi-mode limit cycle oscillations[C]// International Conference on Ship and Offshore Technology, ICSOT India 2015. Kharagpur, India: Royal Institution of Naval Architects, 2015: 127-134.

[3] ECKHOFF K, MUREN J. Deep water TLPs - tether system loading[C]// Proceedings of the 1998 8th International Offshore and Polar Engineering Conference. Part 2 (of 4). Montreal, Can: ISOPE, 1998: 168-177.

[4] D'SOUZA R, AGGARWAL R. The tension leg platform technology - Historical and recent developments[C]// OTC Brasil 2013 - From North to South: A Wealth of Opportunities. Rio de Janeiro, Brazil: OTC, 2013: 1899-1916.

[5] PALLINI Jr J, WALD G, BOZEMAN J D. Slip nut top tendon connection[C]// Offshore Technology Conference. Houston, Texas: Offshore Technology Conference, 1990: 6447-6454

[6] PALLINI J W, YU A. TLP tendon connections top and bottom terminations and tendon pipe couplings $[\mathrm{C}] / /$ Proceedings of the 16th Annual Energy - Sources Technology Conference and Exhibition. Houston, TX, USA: Publ by ASME, 1993: 111-118.

[7] BOYUM E. Analyses of top connectors for a TLP using ADINA[J]. Computers and Structures, 1995, 56(2-3): 269-281.

[8] D'ANGELO L, VITERI M, BRADBERRY R, et al. Technology qualification of tendon connectors for deep and ultra deep water application[C]// OTC Brazil Offshore TechnoogyConference. Rio de Janeiro, Brazil: OTC, 2015: 1-9.

[9] LI Songyu, WANG Liquan, YAO Shaoming, et al. Modelling, simulation and experiment of the spherical flexible joint stiffness[J]. Mechanical Sciences, 2018, 9(1): $81-89$ 
[10] TIMERMAN G J, CAMPOS M A D, NISHIMOTO K, et al. Coupled dynamic and static analysis of typhoon TLP accident during extreme environmental conditions $[\mathrm{C}] / /$ ASME 2008. Estoril, Portugal: International Conference on Offshore Mechanics and Arctic Engineering. 2008: 713-722.

[11] 王一江, 王香, 王立权, 等. 回火温度对高强海洋平台 钢组织和性能的影响 $[\mathrm{J}]$. 金属热处理, 2018, 43(1):

221-226

WANG Yijiang, WANG Xiang, WANG Liquan, et al. Effect of tempering temperature on microstructure and properties of high-strength offshore platform steel[J]. Heat Treatment of Metals，2018，43(1): 221-226

[12] HOU Tunghsu, HU Weichung. An integrated MOGA approach to determine the Pareto-optimal kanban number and size for a JIT system[J]. Expert Systems with Applications, 2011, 38(5): 5912-5918.

[13] 运飞宏, 王立权, 刘军, 等. 深水卡爪式连接器密封优 化分析及试验研究[J]. 华中科技大学学报, 2017,45(4): 23-28, 56.

YUN Feihong, WANG Liquan, LIU Jun, et al. Optimization analysis and experimental research on seal of subsea collet connector[J]. Jounal of Huazhong University of Science and Technology, 2017, 45(4): 23-28, 56.

[14] 王永菲, 王成国. 响应面法的理论与应用 $[\mathrm{J}]$. 中央民族 大学学报, 2005, 14(3): 236-240.

WANG Yongfei, WANG Guocheng. The application of response surface methodology[J]. Journal of the Central
University for Nationalities，2005， 14(3): 236-240.

[15] 张春宜, 宋鲁凯, 费成巍, 等. 柔性机构动态可靠性分 析的先进极值响应面方法[J]. 机械工程学报, 2017, 53(7): 47-54.

ZHANG Chunyi, SONG Lukai, WEI Chengwei, et al. Advanced extremum response surface method for dynamic reliability analysis on flexible mechanism[J]. Journal of Mechanical Engineering, 2017，53(7): 47-54.

[16] 吕辉, 于德介, 谢展, 等. 基于响应面法的汽车盘式制 动器稳定性优化设计 [J]. 机械工程学报, 2013, 49(9): $55-60$.

LÜ Hui, YU Dejie, XIE Zhan, et al. Optimization of vehicle disc brakes stability based on response surface method[J]. Journal of Mechanical Engineering, 2013, 49(9): 55-60.

[17] HERENCIA J E, HAFTKA R T, BALABANOV V. Structural optimization of composite structures with limited number of element properties[J]. Structural and Multidisciplinary Optimization, 2013 , 47(2): 233-245.

[18] CAIRES S, STERL A. 100-year return value estimates for ocean wind speed and significant wave height from the ERA-40 data[J]. Journal of Climate, 2005, 18(7): 1032-1048.

作者简介: 王立权(通信作者), 男, 1957 年出生, 博士, 教授, 博士研 究生导师。主要研究方向为水下作业智能作业装备技术和海洋工程装备 技术。

E-mail: wangliquan@hrbeu.edu.cn

李松羽, 男, 1989 年出生, 博士。主要研究方向为海洋工程装备。

E-mail: lisongyu@hrbeu.edu.cn 